\title{
Characterization of Dental Bonded Interface Degradation Using Focused Ion Beam and High-Resolution Transmission Electron Microscopy
}

\author{
Duarte, S Jr*, A. Avishai**, Sadan A* \\ *Department of Comprehensive Care, Case Western Reserve University, 10900 Euclid Ave, \\ Cleveland, $\mathrm{OH}, 44106$ \\ **Department of Materials Science and Engineering, Case Western Reserve University, 10900 \\ Euclid Ave, Cleveland, OH, 44106
}

Esthetic bonded restorations are the most popular category of dental restoration. In order to promote adequate bond strengths, resin monomers must interlock micromechanically with the exposed collagen network of dental structures. Human dentin must be etched or modified by the use of etchants or acidic monomers, before the application of resin monomers. Microchannels are created by the action of the etchants, and the monomers must flow into these channels of nanometric dimensions forming a polymer and collagen network called "hybrid layer" [1]. Remaining water in the collagen network can dilute or modify the monomer, reducing the ability to entirely fill and seal the etched surface. Therefore, porosity within the hybrid layer poses a problem, because of retained water [2]. To elucidate the characteristics of the resin-impregnated layer, long-term investigation is needed.

The aim of this study was to evaluate the application of focused ion beam (FIB) and high-resolution transmission electron microscope (TEM) on the hybrid layer. The specimens were evaluated immediately after bonding and after 1 year of water storage at $37^{\circ} \mathrm{C}$. Human dentin was exposed and restored with ethanol- and water-based dentin adhesive (Single Bond Plus, 3M ESPE) followed by resin composite restoration (Filtek Z250, 3M ESPE). The specimens were immersed in 50\% ammoniacal silver nitrate dye to trace possible voids, or porosity within the hybrid layer. The specimens were fixed in $2.0 \%$ paraformaldehyde $/ 2.5 \%$ glutaraldehyde in $0.2 \mathrm{M}$ sodium cacodylate buffer, followed by demineralization in $6 \mathrm{~N} \mathrm{HCl}$, and deproteinization in $2 \% \mathrm{NaOCl}$ to remove the collagen so as to expose the resin network structure. The specimens were then dehydrated in ethanol and dried in hexamethyldisilazane. All specimens were mounted on aluminum stubs and sputter-coated with carbon. The TEM specimen preparation was carried out using a dual focused ion beam (FIB, Nova Nanolab 200, FEI Company) and is illustrated in Fig. 1. Using the electron imaging a specific area of the hybrid layer was identified and targeted for the investigation. To avoid damaging the surface by the ion beam, an initial Pt layer was deposited using the electron beam then followed by a thicker ion beam deposition (Fig 1a). Conventional FIB lift-out sample preparation was used to extract the lamella and thin it to perforation (Fig 1a-1c). During the thinning the electron beam imaging allowed to insure the lamella retained a cross section of the hybrid layer. The hybrid layer was observed under high-resolution field-emission transmission electron microscope (Tecnai F30, FEI Company) operated at 300kV (Fig 2 a,b). The presence of silver deposits was confirmed using EDS analysis. Silver deposits were found throughout the hybrid layer, revealing the interstices among the collagen fibers. A comparison between aged and non-aged specimens showed a possible hydrolytic degradation of the resin network. Conventional TEM sample preparation of decalcified specimens requires the specimen to be embedded in epoxy resin, and then sectioned using an ultramicrotome. 
Since epoxy resin infiltrates the decalcified surface, careful interpretation of the data is needed to avoid misinterpretation. FIB preparation of biological samples does not require any additional steps other than conventional SEM preparation.

The present work shows that the use of FIB and TEM are a sine qua non for investigation of the bonded interface in human teeth. Specifically, FIB preparation of non-embedded, decalcified specimens is a novel approach for the study of resin-bonded interfaces, yielding results not obtainable by any other methodology.

[1] Nakabayashi N, Nakamura M, Yasuda N. Hybrid layer as dentin bonding mechanism. $J$ Eshtet Dent (1991);3(4):133-138.

[2] Sano H, Yoshiyama M, Ebisu S, Burrow MF, Takatsu T, Ciucchi B, et al. Comparative SEM and TEM observations of nanoleakage within the hybrid layer. Oper Dent (1995);20:160-167.

a)

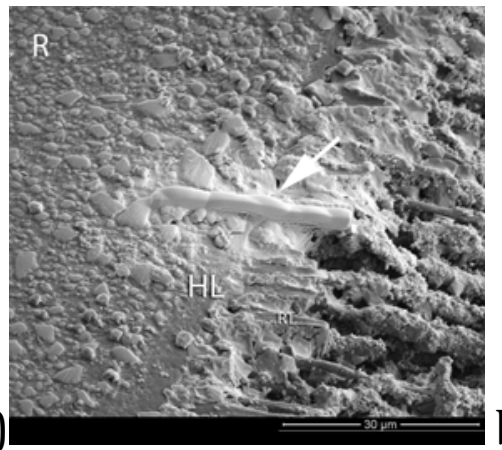

b)

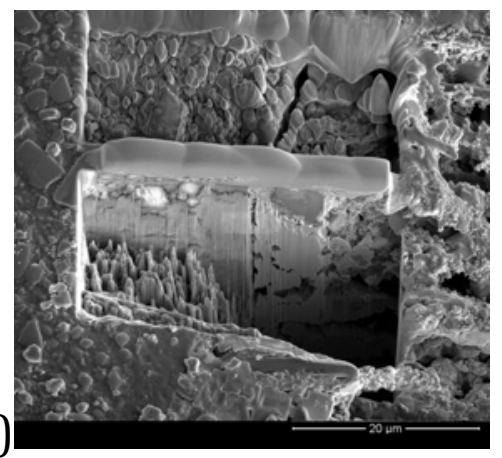

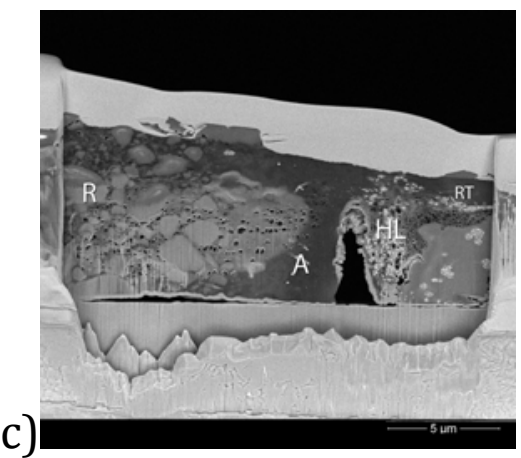

Fig 1: SEM images showing a TEM preparation of dentin adhesive interface using FIB. a) selected area of hybrid layer with Pt deposit (arrow), b) lamella from the selected area ready for lift-out, c) side view of the TEM lamella during thinning, (R:resin, HL: hybrid layer, RT: resin tags, A: adhesive layer).
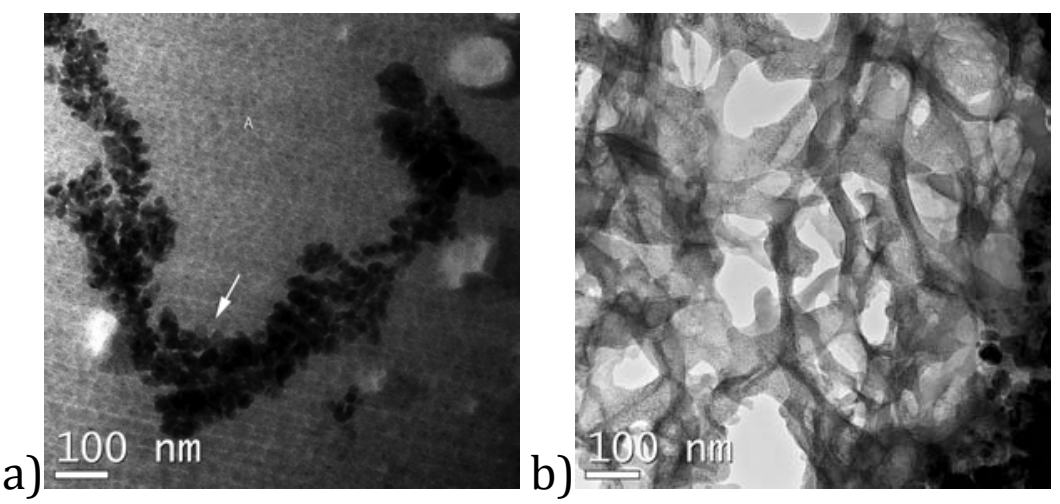

Fig 2: TEM images showing a) a silver-deposit within the adhesive layer, b) a non-embedded decalcified hybrid layer showing the polymer network. 